\title{
Correction to: Immediate and delayed hypersensitivity after intra-arterial injection of iodinated contrast media: a prospective study in patients with coronary angiography
}

\author{
Kyoung-Hee Sohn ${ }^{1,2} \cdot$ Gun-Woo Kim ${ }^{2,3}$. Suh-Young Lee ${ }^{2,4,5} \cdot$ Hyo-Soo Kim ${ }^{6} \cdot$ Sang-Heon Cho ${ }^{2,4,5}$ • Jung-Kyu Han ${ }^{6}$. \\ Hye-Ryun Kang ${ }^{2,4,5}$
}

Published online: 18 February 2020

(C) European Society of Radiology 2020

\section{Correction to: European Radiology (2019) 29:5314-5321} https://doi.org/10.1007/s00330-019-06138-3

The original version of this article, published on 01 April 2019, unfortunately contained a mistake. The presentation of Fig. 1 was incorrect. The corrected figure is given below.

The online version of the original article can be found at https://doi.org/ 10.1007/s00330-019-06138-3

Jung-Kyu Han

hpcrates@gmail.com

$\triangle$ Hye-Ryun Kang

helenmed@snu.ac.kr

1 Department of Internal Medicine, Kyung Hee University Medical Center, Seoul, South Korea

2 Institute of Allergy and Clinical Immunology, Seoul National University Medical Research Center, Seoul, South Korea
Department of Internal Medicine, St. Carollo General Hospital, Suncheon, South Korea

4 Division of Allergy and Clinical Immunology, Department of Internal Medicine, Seoul National University College of Medicine, 101 Daehak-ro, Chongno-gu, Seoul 110-744, South Korea

5 Seoul National University Hospital Regional Pharmacovigilance Center, Seoul, South Korea

6 Department of Internal Medicine and Cardiovascular Center, Seoul National University Hospital, 101 Daehak-ro, Chongno-gu, Seoul 110-744, South Korea 
Fig. 1 a Rate of hypersensitivity reactions according to previous

exposure to intravenous and intraarterial contrast agents: Previous exposure to ICM via intra-arterial route increased the incidence of immediate HSR. b Pie chart showing severity of immediate and delayed hypersensitivity reactions in immediate and delayed HSR. HSR hypersensitivity reactions, ICM iodinated contrast media a

No exposure

- Previous IV exposure
$P=.04$

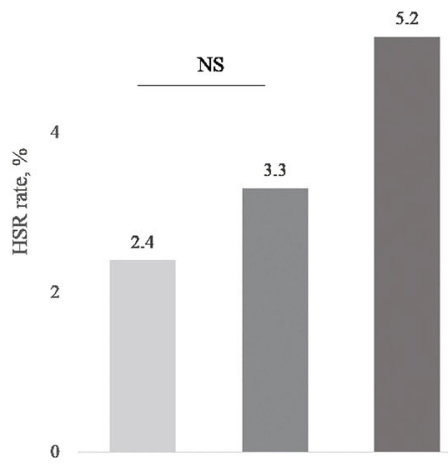

Immediate
- Previous IA exposure

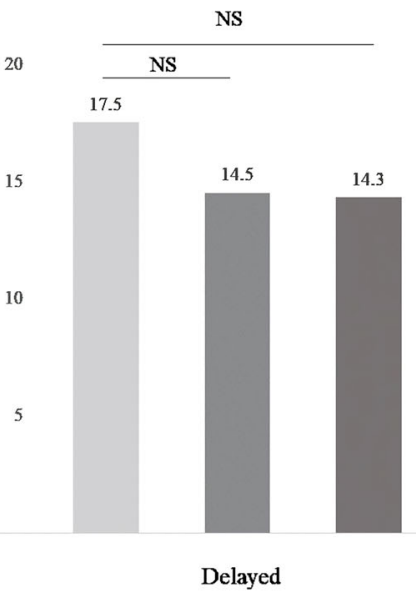

b

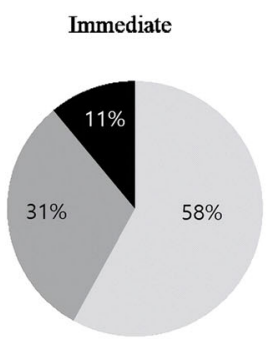

Gr 1

I $\mathrm{Gr} 2$

$85 \%$

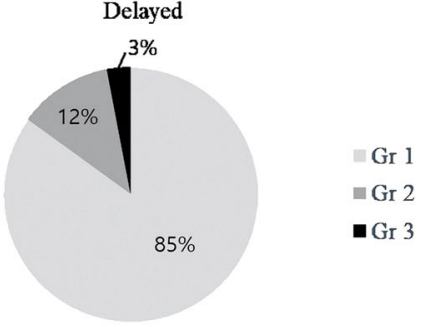

